\title{
Backchannel Responses as Misleading Feedback in Intercultural Discourse
}

\author{
Han Z. Li
}

This study examined the relationship between the frequency of backchannel responses and listener recall scores in inter- and intra-cultural conversations. The nature of the study was a simulated physician-patient interaction. Participants were 40 Canadians and 40 Chinese who formed 40 dyads in four experimental conditions: Canadian physician/Canadian patient, Chinese physician/Chinese patient, Chinese physician/ Canadian patient, and Canadian physician/Chinese patient. All conversations were video-taped and micro-analyzed. The data generated three intriguing findings. (1) There were significant positive correlations between backchannel responses and listener recall scores in the two intra-cultural groups, indicating that backchannel responses facilitated content communication. (2) There were significant negative correlations between backchannel responses and listener recall scores in the two inter-cultural groups, indicating that backchannel responses might have served as misleading feedback and caused mis-communication. (3) The Chinese/Chinese condition had the highest backchannel responses; the Canadian/Canadian condition had the lowest with the two inter-cultural groups in between. This finding provides support for the Communication Accommodation theory. Implications for verbal and nonverbal communication training in intercultural interactions were discussed.

Keywords: Backchannel Responses; Intercultural Communication; Nonverbal Communication; Communication Accommodation Theory; Intercultural Training

\footnotetext{
Han Z. Li (PhD University of Victoria, 1994) is an Associate Professor at the University of Northern British Columbia. Correspondence to: Han Z. Li, Department of Psychology, University of Northern British Columbia, 3333 University Way, Prince George, BC V2N 4Z9, Canada. Tel: 250-960-6502; Fax: 250-960-5536; Email: lih@unbc.ca. This research was in part supported by a grant from the Social Sciences and Humanities Research Council of Canada. The author would like to thank Dr Janet Bavelas, for consultation in designing and conducting the experiment; Dr Michael Bond, for enlightening suggestions on the design of the experiment; the coders for coding the data and all the participants, for their time and efforts in making this study possible. The author would like to thank Editor James Neuliep and the two anonymous reviewers for constructive comments on an early version of the manuscript.
} 
Backchannel responses refer to the short utterances (e.g., uh, huh, okay, Yeah, I see) that occur in the backchannel by the non-primary speaker or the listener when the front channel is occupied by the primary speaker (Yngve, 1970). Later, scholars extended backchannel responses to include sentence completions, requests for clarification, brief statements, and non-verbal responses (Duncan \& Niederehe, 1974; Duncan \& Fiske, 1977). To distinguish backchannel responses, two conditions must to be met: (a) they do not disturb the primary speaker's speakership, and (b) they do not intend to take over the floor of the current speaker (Clancy, Thompson, Suzuki \& Tao, 1996). The two main functions of backchannel responses are "continuers" and "assessments" (Goodwin, 1986; Schegloff, 1982). That is to say, they are indications to the current speaker that the listener is paying attention to and/or understands what is being said.

Past research on backchannel responses comparing Mandarin-speaking Chinese and other language speakers is limited in that only patterns of backchannel responses were studied, not the relationship between backchannel responses and content communication. This study extended previous research by examining whether backchannel responses facilitated or hindered information communication in intraand inter-cultural conversations. In the following sections, literature on backchannel responses involving Mandarin speakers or comparing Mandarin speakers with English speakers was reviewed.

Liu (1987) found that the frequency of backchannel responses were lower among Chinese than Japanese speakers. Mizuno (1988) also reported that Chinese used backchannel responses less frequently than Japanese. Comparing English with Mandarin speakers, Tao and Thompson (1991) reported the following: (1) there was a higher frequency of backchannel responses among the English speakers than the Mandarin speakers (25\% vs. $8 \%$ ); (2) there were more overlapping backchannel responses among the English than the Chinese speakers (51\% vs. 0\%); (3) 19\% of the backchannel responses among the English speakers functioned as "continuers," whereas none of the backchannel responses among the Mandarin speakers served as "continuers," rather, they served as indications of understanding, confirmation or agreement. In another study, Tao and Thompson found that native Chinese who were fluent in English had the tendency to switch code, using English backchannel responses when conversing in Mandarin. The bilinguals also used more backchannel responses than the Mandarin monolinguals.

Clancy et al. (1996) studied backchannel responses in three linguistic groups although the term "reactive token" was used. Several types of reactive token were distinguished: backchannels (display of interest or understanding), collaborative finishes (when the listener finishes the primary speaker's utterance), repetitions (the listener repeats a portion of the primary speaker's utterance), resumptive openers (a non-lexical element used at the beginning of a turn), and reactive expressions. Drawing data from eight transcripts in three languages, English, Japanese and Mandarin, they found that the amount of speaker change per total number of intonation units was similar in the three language speakers: $28 \%$ in Japanese, $33.6 \%$ 
in English and 28.7\% in Mandarin. But the range of variations and the ratio of reactive tokens to speaker change were greater in English and Japanese than in Mandarin. Furthermore, the Japanese speakers had the highest percentage of backchannels (68.3\%), followed by Mandarin speakers (47.2\%) and the English speakers $(37.9 \%)$. In terms of repetition, the Mandarin speakers $(5.8 \%)$ had higher percentages than the Japanese (2.2\%) and English speakers (1.3\%). In terms of reactive expressions, the English (34.2\%) and the Mandarin speakers (31.1\%) had higher percentages than the Japanese (17.0\%).

\section{Communication Accommodation Theory (CAT)}

CAT states that interlocutors have a tendency to converge or diverge their linguistic codes in their conversations (Giles, Taylor, \& Bourhis, 1973; Giles, Mulac, Bradac, \& Johnson, 1987; Giles \& Smith, 1979). Studies testing CAT in Chinese/Canadian conversations are scarce. Li (2001) found that in their interactions, Chinese participants switched their usual co-operative interruption style to the more intrusive interruption style of the Canadians, an indication that speech convergence occurred. In another study, Li (2004) found that Chinese gazed more frequently when conversing with Canadians than with Chinese, documenting a convergence in intercultural nonverbal behaviour.

Guided by CAT, and building upon previous research, the present study proposed three research questions. The rationale for posing the research questions rather than hypotheses was that the number of studies involving Mandarin speakers was small and the findings were inconsistent, therefore not sufficient to generate any meaningful hypothesis. The research questions were: (1) what were the frequencies of backchannel responses in the four conditions? (2) What were the characteristics of backchannel responses in the four conditions? (3) Did backchannel responses facilitate or hinder information communication?

\section{Method}

\section{Participants}

Eighty-four participants volunteered (no incentives were offered) to participate in this study, of which 44 were males and 40 were females. The participants formed 42 same-gender dyads, two of which were dropped from data analysis because they did not follow the instructions. Participants were third-year, fourth-year or graduate students from a western University in Canada. The majority of the participants were in their twenties or earlier thirties with an average age of 29.1. The mean ages for the Chinese and the Canadian participants were 30.0 and 28.2, respectively. These means were not statistically significant, $t(1,78)=-1.90, p>0.05$. Participants were recruited in classrooms, the university cafeterias and graduate student' offices in 
various departments of the University. Prior to the recruitment of participants, ethics approval was obtained from the University's Ethics Review Board.

Of the 80 participants, 40 were Chinese (20 males and 20 females) speaking Mandarin Chinese as their first language, and 40 were Caucasian Canadians (20 males and 20 females), speaking English as their first language. All Chinese participants grew up in Mainland China and were studying at a western University in Canada. At the time of the experiment, the Chinese participants had been in Canada for an average of 27 months, the range being 0.5-60 months. Care was taken to ensure that the Chinese participants had sufficient English language ability (as measured by peer evaluation, self-evaluation and referenced by scores on the Test of English as a Foreign Language (TOEFL) to participate in the conversations. All Chinese participants had a TOEFL score of 575 or above at the time of the experiment.

\section{Experimental Design and Procedures}

A between-subject design was used for the four experimental conditions: Canadian physician/Canadian patient, Chinese physician/Chinese patient, Chinese physician/ Canadian patient, and Canadian physician/Chinese patient. According to the time of their availability, participants were either paired with a partner from their own culture (Canadian physician/Canadian patient or Chinese physician/Chinese patient) or one from a different culture (Chinese physician/Canadian patient or Canadian physician/Chinese patient). All dyads were same-gender; that is, males were paired with males and females with females.

All dyads (10 in each of the four experimental conditions) engaged in the same communication task, which involved simulating a physician-patient interview. The experiment had two phases. In the first phase, the patient was given a simple case history to study; then, during the experimental session, he/she presented the case history to the physician. The case history was developed in Chinese, translated into English and then translated back into Chinese to check for accuracy. The Chinese physician/Chinese patient condition used the Chinese version, while the other three conditions used the English version ( $\mathrm{Li}, 1999 \mathrm{~b}$ ).

Upon arrival to the lab, and after the role of patient or physician was randomly assigned by a draw, the patient was given sufficient time to study the case history. A multiple-choice test (as a manipulation check) was then given to the patient to ensure that he/she had mastered the content. Meanwhile, the physician was given a list of information which he/she should obtain from the patient during the conversation. The list of information was relevant to the physician-patient interview in general (e.g., what the exact problem was; whether the patient had a previous occurrence of the problem), and not specific to the content of the case history. The physician was also instructed to feel free to ask the patient questions during the conversation. After this role-play, the physician completed an open-ended test designed to examine how much information was successfully communicated from the speaker to the listener. 
In the second phase, the participant playing the role of physician was first given information on the use of codeine (taken from Compendium of Pharmaceuticals and Specialties; Canadian Pharmaceuticals Association, 1982), which was appropriate for the case history just presented. After studying the information, the physician took a multiple-choice test to ensure that he or she had adequately mastered the content. The physician then informed the patient about the use of codeine. Immediately after the conversation, the participant playing the role of the patient took an open-ended test, which measured how much information about the medication was successfully communicated.

In performing the dialogues, the following guidelines were followed. (1) Neither physician nor patient was given specific instructions regarding how to pass the information. That is, the physician was not given a set format for asking questions or explaining codeine, and the patient was not told how to tell his or her case history or what to ask about the medication. (2) To minimize memory errors, both patient and physician had their information sheet available during the interaction but were not to read from it or show it to the other. (c) Participants were also instructed to convey the information in a natural, "talking" manner. All conversations were video-taped with the informed consent of the participants.

\section{Coding of Backchannel Responses}

The video-tapes were made using three high-resolution cameras, two in zoom and the third in normal mode. The two zoom-mode cameras filmed a split screen closeup of images of both participants side by side; the normal-mode camera filmed a full screen of the two participants facing each other. All three screens were synchronized into one picture on the TV screen, with the split screen on top of the full screen. A high-resolution TV/VCR was used to score backchannel response activities.

The operational definition of a backchannel response is any verbal or nonverbal (nod only, not smile) act occurring during the conversation in a non-intrusive manner (not interrupting the speech turn of the current speaker). It can be presented as one word (e.g., Yeah) or a statement (e.g., Oh, I see) or a question (e.g., Is that so). If it is posed as a question, it is judged as a backchannel response if its tone is falling, indicating that an answer from the current speaker is not required. If the question is presented in a rising tone, it is an indication that a response from the current speaker is requested. It is then judged as a question in the front channel, not a backchannel response. Examples of backchannel responses are presented in Appendix A.

Three trained research assistants, one English-speaking and two bilinguals, made verbatim transcripts of the videotaped conversations. The transcription technique was identical to that of Beaumont and Cheyne (1998) and Kollock, Blumstein and Schwartz (1985). Although transcripts were available, coders were required to code backchannel responses from the videotape, using the transcripts as references. Prior to coding the data, coders participated in a training session with the following instructions: (1) read the criteria at least twice; (2) watch the tape while reflecting on 
coding criteria; (3) code for the first time by watching the tape and listening to the dialogue; (4) code for the second time by focusing on non-verbal activities.

Following the training session, the coders worked together to decide on the backchannel categories. Once the categories were determined, the three coders independently scored $15 \%$ of the data. Inter-coder reliability (Pearson correlation) was 0.86 and 0.89 . Differences were reconciled by going through the coding standards and viewing the video-tape together. In coding the data, coders were required to mark for overlapping and non-overlapping backchannel responses on the transcripts.

\section{Scoring of Listener Recall and Speaker Presentation}

First, an answer key was developed for the two open-ended tests. The first test (for Dialogue 1) consisted of 10 open-ended questions (see Appendix B). Questions 1-5, 7 and 8 were each assigned 4 points; Question 6 was assigned 12 points, and Questions 9 and 10 were each assigned 8 points. The total points for Test 1 (for Dialogue 1) were 56. The second test (for Dialogue 2) was made up of seven openended questions. Questions 1, 5, 6 and 7 were each assigned 4 points; Question 2 was assigned 28 points, Question 3 was assigned 8 points and Question 4 was assigned 16 points. The total points for Test 2 (for Dialogue 2) were 68.

The points for each question were allocated on the basis of the number of information units. The smallest string of words with meaning was the unit of analysis (Bales, 1950; Li, 1999a; Roter \& Hall, 1992). Each unit of information was worth 4 points. Some questions required answers containing one unit of information while others contained several units of information. Thus some questions were valued at 4 points, while others were as high as 28 points. For example, the answer to Question 1 in Test 1 "Why did the patient come to see you?" only contained one unit of information: chest pains. Whereas answers to Question 2 in Test 2 "What are the possible side effects after you take codeine?" contained seven units of information: drowsiness, nausea, vomiting, constipation, an increase in heart rate, agitation and respiratory problems. Thus Question 2 in Test 2 was worth 28 points.

For each question, one or several correct answers were provided. If the answer was essentially identical to the key, it was scored as 4 . If the answer was very close to the correct answer, it was scored as 3. If the answer was related to the correct answer (e.g., describing but not naming), it was scored as 2 . If the answer was remotely related to the correct answer in that the meaning could be inferred, it was scored as 1 . Blank or wrong answers were scored as 0 . A scale of 0-4 allowed the scorer to assign an appropriate rating to the range of answers given.

For example, Question 7 in Test 1 was: "What was the main reason the patient went swimming?" If the answer was "the reason the patient went swimming was to exercise his/her legs", it was scored as 4 . If the answer was essentially "swimming is good for his/her legs", it was scored as 3. If the answer was essentially "to do exercise because the patient has difficulties walking", it was scored as 2 . 
The listener recall scores were based on a test the listeners took immediately after each dialogue. The speaker presentation scores were based on video-tapes applying the same scoring standards as those for the listener recall.

\section{Results}

The mean time to complete the first dialogue was 4 minutes for the Canadian/ Canadian condition (240 seconds); 5 minutes and 18 seconds for the Chinese/ Chinese condition (318 seconds), 5 minutes and 40 seconds for the Chinese physician/Canadian patient condition (340 seconds) and 4 minutes and 57 seconds for the Canadian physician/Chinese patient condition (297 seconds). Univariate $F$-test showed that no two groups were significantly different from each other, $F(3,36)=0.81, p>0.05, \eta^{2}=0.06$.

The mean time to complete the second dialogue was 3 minutes and 47 seconds for the Canadian/Canadian condition (227), 4 minutes and 35 seconds for the Chinese/ Chinese condition (275 seconds), 3 minutes and 13 seconds for the Chinese physician/Canadian patient condition (193 seconds) and 3 minutes and 55 seconds for the Canadian physician/Chinese patient condition (235 seconds). Univariate F-test showed that no two groups were significantly different from each other, $F(3,36)=1.1, p>0.05, \eta^{2}=0.08$.

\section{Frequency of Backchannel Responses (RQ1)}

\section{Dialogue 1}

As shown in Table 1, the Canadian physician/Canadian patient group had the lowest speaker backchannel responses; the Chinese physician/Chinese patient group had the highest, with the two inter-cultural groups in between. The difference between the Canadian/Canadian and Chinese/Chinese groups reached statistical significance, $t(1,18)=2.34, p<0.05, \eta^{2}=0.23$. The difference between the Canadian/Canadian and Chinese physician/Canadian patient groups also reached statistical significance, $t(1,18)=2.19, p<0.05, \eta^{2}=0.21$. The difference between the Canadian/Canadian and Canadian physician/Chinese patient groups did not reach statistical significance, $t(1,18)=1.58, p>0.05, \eta^{2}=0.12$. The difference between the Chinese/Chinese and Canadian physician/Chinese patient groups did not reach statistical significance, $t(1,18)=1.33, p>0.05, \eta^{2}=0.09$. The difference between the Chinese/Chinese and Chinese physician/Canadian patient groups did not reach statistical significance, $t(1,18)=0.7, p>0.05, \eta^{2}=0.03$. The difference between the Canadian physician/ Chinese patient and Chinese physician/Canadian patient groups did not reach statistical significance, $t(1,18)=0.75, p>0.05, \eta^{2}=0.03$.

For the listener backchannel responses, the four groups were similar, $F(3,36)=0.08, p>0.05, \eta^{2}=0.007$. Also shown in Table 1 , the four groups had similar speaker presentation scores but very different listener recall scores. Detailed analyses of speaker presentation and listener recall scores are presented elsewhere (Li, 1999a). 
106 H. Z. Li

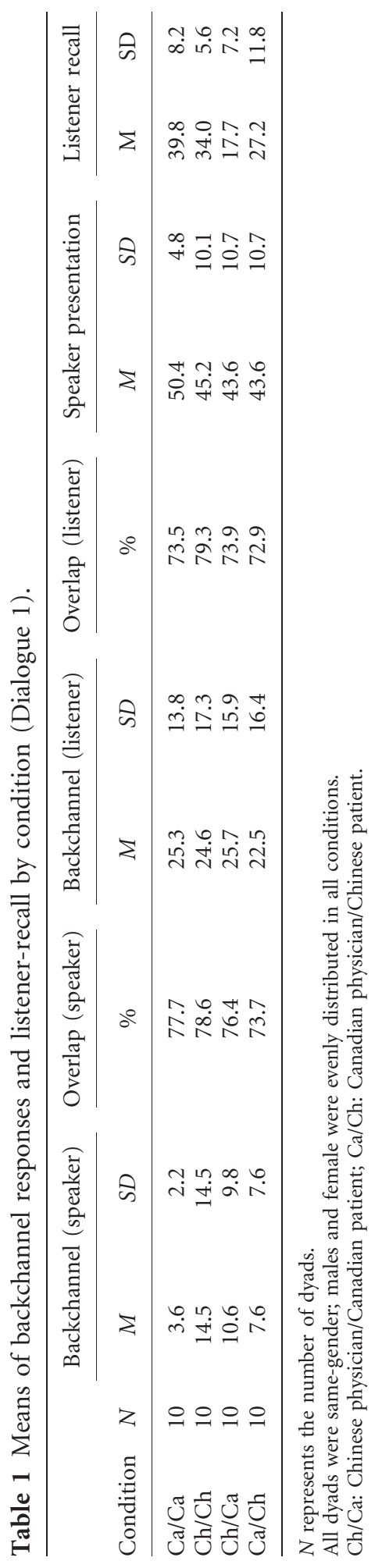


Dialogue 2

As shown in Table 2, the Canadian physician/Chinese patient group had the highest speaker backchannel responses; the Chinese physician/Canadian patient group had the lowest, with the remaining two groups in between. However, the group differences did not reach statistical significance, $F(3,36)=0.09, p>0.05, \eta^{2}=0.09$. For the listener backchannel responses, the Chinese/Chinese group had the highest scores in comparison with the other three groups, but these differences also did not reach statistical significance, $F(3,36)=1.31, p>0.05, \eta^{2}=0.10$.

\section{Characteristics of Backchannel Responses (RQ2)}

Overlapping vs. non-overlapping

As can be seen in Table 3, the majority of backchannel responses are overlapping, that is, non-verbal and verbal responses were elicited simultaneously. In Dialogue 1, the Chinese physicians had higher percentages of overlapping backchannel responses than the other three groups. On the other hand, the Chinese patients in the Canadian physician/Chinese patient condition had lower percentages of overlapping backchannel responses than the other three groups.

In Dialogue 2, the Canadian physicians in the Canadian/Canadian condition had the lowest percentages of overlapping backchannel responses; the Canadian physicians in the Canadian physicians/Chinese patient condition had the highest percentages of overlapping backchannel responses, with the other two conditions in between. For patient backchannel responses, the Chinese physician/Canadian patient condition had lower percentages than the other three conditions.

\section{Categories of backchannel responses}

Tables 4-7 present the 14 categories of backchannel responses for the four experimental conditions respectively. As can be seen in Tables 3 and 4, in both the Canadian/Canadian and Chinese/Chinese conditions, the majority of backchannel responses were in the categories of "Nod", "Nod with OK", and "Nod with Uhm". Canadians used "Nod with Yeah" and "Okay" more frequently than Chinese whereas Chinese used "Uhm", "Right" and "Repeat" more often than Canadians. The Chinese also had a special category "Is that so" (Shi Ma), which the Canadians did not have.

In the Canadian physician/Chinese patient condition, the Canadians exhibited higher proportions of "Uhm" than the Canadians did in the Canadian/Canadian condition. The Chinese in the Canadian physician/Chinese patient condition exhibited lower proportions of "Right" than the Chinese in the Chinese/Chinese condition.

In the Chinese physician/Canadian patient condition, the Canadians exhibited higher proportions of "Right" than the Canadians did in the Canadian/Canadian condition. The Chinese exhibited lower proportions of "Uhm" than the Chinese did in the Chinese/Chinese condition. 


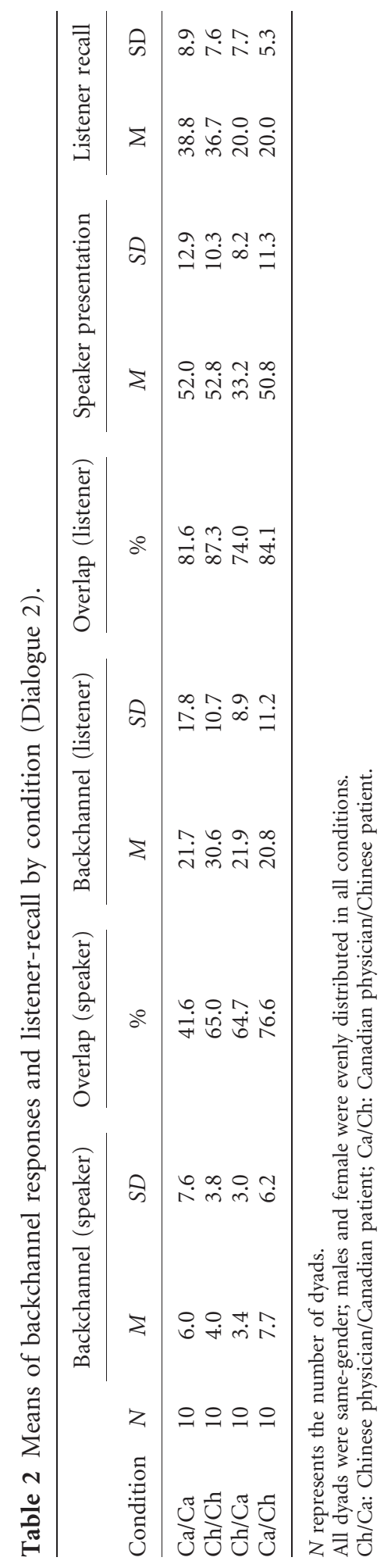


Table 3 Frequency and percentage of overlapping backchannel responses by condition.

\begin{tabular}{|c|c|c|c|c|c|c|c|c|c|}
\hline \multirow[b]{3}{*}{ Condition } & \multirow[b]{3}{*}{$N$} & \multicolumn{4}{|c|}{ Dialogue 1} & \multicolumn{4}{|c|}{ Dialogue 2} \\
\hline & & \multicolumn{2}{|c|}{ Frequency } & \multicolumn{2}{|c|}{$\%$} & \multicolumn{2}{|c|}{ Frequency } & \multicolumn{2}{|c|}{$\%$} \\
\hline & & Dr. & Pt. & Dr. & Pt. & Dr. & Pt. & Dr. & Pt. \\
\hline $\mathrm{Ca} / \mathrm{Ca}$ & 10 & $186 / 253$ & $28 / 36$ & 73.5 & 77.8 & $25 / 60$ & $177 / 217$ & 41.6 & 81.6 \\
\hline $\mathrm{Ch} / \mathrm{Ch}$ & 10 & $195 / 246$ & $114 / 145$ & 79.3 & 78.6 & $26 / 40$ & $267 / 306$ & 65.0 & 87.3 \\
\hline $\mathrm{Ch} / \mathrm{Ca}$ & 10 & $190 / 257$ & $81 / 106$ & 73.9 & 76.4 & $22 / 34$ & $162 / 219$ & 64.7 & 73.9 \\
\hline $\mathrm{Ca} / \mathrm{Ch}$ & 10 & $164 / 225$ & $56 / 76$ & 72.9 & 73.7 & $59 / 77$ & $175 / 208$ & 76.6 & 84.1 \\
\hline
\end{tabular}

$N$ represents the number of dyads.

All dyads were same-gender; males and female were evenly distributed in all conditions.

Ch/Ca: Chinese physician/Canadian patient; Ca/Ch: Canadian physician/Chinese patient.

Table 4 Categories of backchannel responses (Canadian/Canadian condition).

\begin{tabular}{|c|c|c|c|c|c|c|c|c|}
\hline \multirow[b]{3}{*}{ Category } & \multicolumn{4}{|c|}{ Dialogue 1} & \multicolumn{4}{|c|}{ Dialogue 2} \\
\hline & \multicolumn{2}{|c|}{ Speaker } & \multicolumn{2}{|c|}{ Listener } & \multicolumn{2}{|c|}{ Speaker } & \multicolumn{2}{|c|}{ Listener } \\
\hline & Sum & $\%$ & Sum & $\%$ & Sum & $\%$ & Sum & $\%$ \\
\hline Nod & 17 & 47.2 & 102 & 40.3 & 12 & 20.0 & 102 & 47.0 \\
\hline Nod with OK & 5 & 13.9 & 31 & 12.3 & 8 & 13.3 & 33 & 15.2 \\
\hline Nod with Uhm & 1 & 2.8 & 31 & 12.3 & 1 & 1.7 & 15 & 6.9 \\
\hline Nod with Yeah & 2 & 5.6 & 17 & 6.7 & 4 & 6.7 & 4 & 1.8 \\
\hline Nod with I see & 0 & 0.0 & 2 & 0.8 & 0 & 0.0 & 0 & 0.0 \\
\hline Nod with Right & 0 & 0.0 & 1 & 0.4 & 1 & 1.7 & 1 & 0.5 \\
\hline Okay & 3 & 8.3 & 41 & 16.2 & 15 & 25.0 & 36 & 16.6 \\
\hline Yeah & 4 & 11.1 & 2 & 0.8 & 12 & 20.0 & 17 & 7.8 \\
\hline Uhm & 4 & 11.1 & 17 & 6.7 & 6 & 10.0 & 7 & 3.2 \\
\hline I see & 0 & 0.0 & 2 & 0.8 & 0 & 0.0 & 0 & 0.0 \\
\hline Right & 0 & 0.0 & 4 & 1.6 & 1 & 1.7 & 1 & 0.5 \\
\hline $\mathrm{Oh}$ & 0 & 0.0 & 3 & 1.2 & 0 & 0.0 & 1 & 0.5 \\
\hline Repeat & 0 & 0.0 & 0 & 0.0 & 0 & 0.0 & 0 & 0.0 \\
\hline Shi Ma (Is that so) & 0 & 0.0 & 0 & 0.0 & 0 & 0.0 & 0 & 0.0 \\
\hline Total & 36 & 100 & 253 & 100 & 60 & 100 & 217 & 100 \\
\hline
\end{tabular}

\section{Correlations between Backchannel Responses and Listener Recall Scores (RQ3)}

Pearson correlations were calculated between the frequency of backchannel responses and listener recall scores for both Dialogue 1 and Dialogue 2. In performing Dialogue 1 , the correlation between speaker backchannel responses and listener recall scores in the Canadian/Canadian group reached statistical significance, $r(10)=0.81, p<0.01$. The correlation between listener backchannel responses and listener recall scores in the Canadian/Canadian group also reached statistical significance, $r(10)=0.35$, $p<0.05$. None of the other correlations in the other groups reached statistical significance. 
110 H. Z. $\mathrm{Li}$

Table 5 Categories of backchannel responses (Chinese/Chinese condition).

\begin{tabular}{|c|c|c|c|c|c|c|c|c|}
\hline \multirow[b]{3}{*}{ Category } & \multicolumn{4}{|c|}{ Dialogue 1} & \multicolumn{4}{|c|}{ Dialogue 2} \\
\hline & \multicolumn{2}{|c|}{ Speaker } & \multicolumn{2}{|c|}{ Listener } & \multicolumn{2}{|c|}{ Speaker } & \multicolumn{2}{|c|}{ Listener } \\
\hline & Sum & $\%$ & Sum & $\%$ & Sum & $\%$ & Sum & $\%$ \\
\hline Nod & 30 & 20.7 & 36 & 14.6 & 7 & 17.5 & 113 & 36.9 \\
\hline Nod with OK & 11 & 7.6 & 4 & 1.6 & 5 & 12.5 & 10 & 3.3 \\
\hline Nod with Uhm & 23 & 15.9 & 59 & 24.0 & 3 & 7.5 & 58 & 19.0 \\
\hline Nod with Yeah & 2 & 1.4 & 1 & 0.4 & 0 & 0.0 & 2 & 0.7 \\
\hline Nod with I see & 1 & 0.7 & 15 & 6.1 & 1 & 2.5 & 5 & 1.6 \\
\hline Nod with Right & 9 & 6.2 & 1 & 0.4 & 2 & 5.0 & 2 & 0.7 \\
\hline Okay & 15 & 10.3 & 1 & 0.4 & 2 & 5.0 & 10 & 3.3 \\
\hline Yeah & 2 & 1.4 & 2 & 0.8 & 1 & 2.5 & 3 & 1.0 \\
\hline Uhm & 25 & 17.2 & 99 & 40.2 & 7 & 17.5 & 96 & 31.4 \\
\hline I see & 0 & 0.0 & 0 & 0.0 & 0 & 0.0 & 0 & 0.0 \\
\hline Right & 16 & 11.0 & 3 & 1.2 & 8 & 20.0 & 0 & 0.0 \\
\hline Oh & 0 & 0.0 & 0 & 0.0 & 0 & 0.0 & 0 & 0.0 \\
\hline Repeat & 8 & 5.5 & 16 & 6.5 & 3 & 7.5 & 6 & 2.0 \\
\hline Shi Ma (Is that so) & 3 & 2.1 & 9 & 3.7 & 1 & 2.5 & 1 & 0.3 \\
\hline Total & 145 & 100 & 246 & 100 & 40 & 100 & 306 & 100 \\
\hline
\end{tabular}

Table 6 Categories of backchannel responses (Chinese physician/Canadian patient).

\begin{tabular}{|c|c|c|c|c|c|c|c|c|}
\hline \multirow[b]{3}{*}{ Category } & \multicolumn{4}{|c|}{ Dialogue 1} & \multicolumn{4}{|c|}{ Dialogue 2} \\
\hline & \multicolumn{2}{|c|}{ Speaker } & \multicolumn{2}{|c|}{ Listener } & \multicolumn{2}{|c|}{ Speaker } & \multicolumn{2}{|c|}{ Listener } \\
\hline & Sum & $\%$ & Sum & $\%$ & Sum & $\%$ & Sum & $\%$ \\
\hline Nod & 33 & 31.1 & 85 & 33.1 & 10 & 29.4 & 65 & 29.7 \\
\hline Nod with OK & 13 & 12.3 & 24 & 9.3 & 1 & 2.9 & 53 & 24.2 \\
\hline Nod with Uhm & 12 & 11.3 & 56 & 21.8 & 1 & 2.9 & 44 & 20.1 \\
\hline Nod with Yeah & 3 & 2.8 & 12 & 4.7 & 6 & 17.6 & 2 & 0.9 \\
\hline Nod with I see & 0 & 0.0 & 6 & 2.3 & 1 & 2.9 & 3 & 1.4 \\
\hline Nod with Right & 2 & 1.9 & 2 & 0.8 & 0 & 0.0 & 5 & 2.3 \\
\hline Okay & 13 & 12.3 & 28 & 10.9 & 4 & 11.8 & 22 & 10.0 \\
\hline Yeah & 14 & 13.2 & 11 & 4.3 & 5 & 14.7 & 5 & 2.3 \\
\hline Uhm & 6 & 5.7 & 14 & 5.4 & 5 & 14.7 & 15 & 6.8 \\
\hline I see & 0 & 0.0 & 0 & 0.0 & 1 & 2.9 & 0 & 0.0 \\
\hline Right & 6 & 5.7 & 1 & 0.4 & 0 & 0.0 & 4 & 1.8 \\
\hline $\mathrm{Oh}$ & 4 & 3.8 & 8 & 3.1 & 0 & 0.0 & 1 & 0.5 \\
\hline Repeat & 0 & 0.0 & 10 & 3.9 & 0 & 0.0 & 0 & 0.0 \\
\hline Shi Ma (Is that so) & 0 & 0.0 & 0 & 0.0 & 0 & 0.0 & 0 & 0.0 \\
\hline Total & 106 & 100 & 257 & 100 & 34 & 100 & 219 & 100 \\
\hline
\end{tabular}

In performing Dialogue 2, the correlation between listener backchannel responses and listener recall scores in the Canadian/Canadian group was significant, $r(10)=0.41$, $p<0.05$. The correlation between listener backchannel responses and listener recall scores in the Chinese/Chinese group was significant, $r(10)=0.50, \quad p<0.05$. 
Table 7 Categories of backchannel responses (Canadian physician/Chinese patient).

\begin{tabular}{|c|c|c|c|c|c|c|c|c|}
\hline \multirow[b]{3}{*}{ Category } & \multicolumn{4}{|c|}{ Dialogue 1} & \multicolumn{4}{|c|}{ Dialogue 2} \\
\hline & \multicolumn{2}{|c|}{ Speaker } & \multicolumn{2}{|c|}{ Listener } & \multicolumn{2}{|c|}{ Speaker } & \multicolumn{2}{|c|}{ Listener } \\
\hline & Sum & $\%$ & Sum & $\%$ & Sum & $\%$ & Sum & $\%$ \\
\hline Nod & 18 & 23.7 & 87 & 38.7 & 18 & 23.4 & 85 & 40.9 \\
\hline Nod with OK & 6 & 7.9 & 34 & 15.1 & 13 & 16.9 & 40 & 19.2 \\
\hline Nod with Uhm & 22 & 28.9 & 56 & 24.9 & 16 & 20.8 & 45 & 21.6 \\
\hline Nod with Yeah & 10 & 13.2 & 2 & 0.9 & 0 & 0.0 & 7 & 3.4 \\
\hline Nod with I see & 7 & 9.2 & 1 & 0.4 & 0 & 0.0 & 3 & 1.4 \\
\hline Nod with Right & 1 & 1.3 & 0 & 0.0 & 4 & 5.2 & 5 & 2.4 \\
\hline Okay & 1 & 1.3 & 20 & 8.9 & 12 & 15.6 & 10 & 4.8 \\
\hline Yeah & 5 & 6.6 & 4 & 1.8 & 0 & 0.0 & 5 & 2.4 \\
\hline Uhm & 5 & 6.6 & 12 & 5.3 & 11 & 14.3 & 7 & 3.4 \\
\hline I see & 0 & 0.0 & 0 & 0.0 & 0 & 0.0 & 1 & 0.5 \\
\hline Right & 1 & 1.3 & 3 & 1.3 & 2 & 2.6 & 0 & 0.0 \\
\hline $\mathrm{Oh}$ & 0 & 0.0 & 1 & 0.4 & 0 & 0.0 & 0 & 0.0 \\
\hline Repeat & 0 & 0.0 & 5 & 2.2 & 1 & 1.3 & 0 & 0.0 \\
\hline Shi Ma (Is that so) & 0 & 0.0 & 0 & 0.0 & 0 & 0.0 & 0 & 0.0 \\
\hline Total & 76 & 100 & 225 & 100 & 77 & 100 & 208 & 100 \\
\hline
\end{tabular}

The correlation between speaker backchannel responses and listener recall scores in the Chinese physician/Canadian patient group was significant, $r(10)=-0.61, p<0.01$, as was the correlation between speaker backchannel responses and listener recall scores in the Canadian physician/Chinese patient group, $r(10)=-0.43, p<0.05$.

\section{Discussion}

\section{Correlations between the Frequency of Backchannel Responses and Listener Recall}

The two most meaningful findings were the significant positive correlations between backchannel responses and listener recall scores in the two intra-cultural conditions and the negative correlations in the two inter-cultural conditions. The former indicates that backchannel responses facilitated content communication, whereas the latter implies that backchannel responses hindered content communication. In intracultural interactions, the more backchannel responses, the better interlocutors transmitted information. The implication is that in order to communicate effectively, intra-cultural interlocutors are encouraged to elicit more backchannel responses.

In intercultural interactions, the situation is more complicated. The negative correlations between backchannel responses and listener recall scores could indicate that backchannel responses may have served as misleading feedback, thus preventing the information from being transmitted correctly. In these instances, it could be argued that the listener may have nodded to show "I am paying attention" but the speaker could have taken this to mean "I understand what you are saying" and continued to the next utterance. This finding has implications for intercultural 


\section{H. Z. Li}

communication training. If intercultural interactants are instructed to "nod" or say " $u h m$ " when they actually understand, and ask for clarification when understanding is in question, information transmission could be improved.

\section{Frequency of Backchannel Responses and CAT}

Significant cultural differences in the frequency of backchannel responses were found. The Chinese/Chinese condition exhibited the highest frequency and the Canadian/ Canadian had the lowest frequency with the two intercultural conditions in between, providing support for CAT in that a backchannel convergence occurred in the intercultural conditions. This finding is in line with previous research ( $\mathrm{Li}, 2001)$ regarding interruption style convergence of the Chinese (co-operative interruption style) to the Canadians (intrusive interruption style) and the frequency of gaze in Chinese/Canadians interactions ( $\mathrm{Li}, 2004)$. It was found that frequencies of gaze and mutual gaze were higher in the Canadian/Canadian conversations and lower in the Chinese/Chinese conversations, but when Chinese and Canadians conversed, the frequency of gaze was similar to that of the Canadian/Canadian condition, a strong indication that the Chinese converged to the gaze style of the Canadians.

The finding that Chinese/Chinese exhibited higher frequencies of backchannel responses than Canadians were in agreement with Clancy et al. (1996) who reported that Mandarin Chinese displayed more backchannel responses than English speakers. However, this finding is in disagreement with Tao and Thompson (1991) who found that Mandarin Chinese had lower frequencies of backchannel responses than English speakers. In terms of repetition, our finding that Chinese used more "repeat" than Canadians seems consistent with previous research (Clancy et al., 1996).

\section{Categories of Backchannel Responses and CAT}

In terms of the categories of backchannel responses, a two-way code switching in the two inter-cultural conditions occurred, providing support for CAT. In the Canadian physician/Chinese patient condition, the Canadians showed more "Uhm" than the Canadians did in the Canadian/Canadian condition. The Chinese in the Canadian physician/Chinese patient condition showed less "Right" than the Chinese in the Chinese/Chinese condition. In the Chinese physician/Canadian patient condition, the Canadians showed more "Right" than the Canadians did in the Canadian/Canadian condition. The Chinese showed more "Uhm" than the Chinese did in the Chinese/ Chinese condition.

These findings are in agreement with previous research. Tao and Thompson (1991) reported that native Chinese who were fluent in English had a tendency to switch code, using English backchannel responses. Heinz (2003) found that German bilinguals had a tendency to converge to American backchannel style even when conversing with their German friends. 


\section{Limitations and Future Research}

An apparent limitation of this study is that it was a simulated physician-patient interaction instead of actual physician-patient interaction. Therefore, the results can not be generalized to actual physician-patient communication. Instead, interpretation of the findings should be focused on the comparison between inter- and intracultural conversations. Future research could sample actual physician-patient interaction in inter- and intra-cultural combinations. The results would be more specific to health communication. For further studies on backchannel response behaviours in inter-cultural communication, samples in natural settings rather than lab settings would provide additional insight.

In conclusion, a major contribution of this study is the finding that backchannel responses functioned differently in relation to content transmission in intra- and intercultural conversations. If intercultural interlocutors can be trained to pay attention to their backchannel responses, they may be less likely to miscommunicate. The findings also provide evident support for CAT, an indication that Communication Accommodation Theory is still robust since its debut three decades ago.

\section{References}

Bales, R. F. (1950). Interaction process analysis. Cambridge, MA: Addison-Wesley.

Beaumont, S. L., \& Cheyne, J. A. (1998). Interruption in adolescent girls' conversations: Comparing mothers and friends. Journal of Adolescent Research, 13, 272-292.

Canadian Pharmaceuticals Association. (1982). Compendium of pharmaceuticals and specialties: The Canadian preference of health professionals. Toronto, Canada: Southam Murray.

Clancy, P. M., Thompson, S. A., Suzuki, R., \& Tao, H. Y. (1996). The conversational use of reactive tokens in English, Japanese, and Mandarin. Journal of Pragmatics, 26, 355-387.

Duncan, S. Jr., \& Fiske, D. W. (1977). Face-to-face interaction. Hillsdale, NJ: Lawrence Erlbaum Associates, Inc.

Duncan, S. Jr., \& Niederehe, G. (1974). On signalling that it's your turn to speak. Journal of Experimental Social Psychology, 10, 234-247.

Giles, H., Mulac, A., Bradac, J. J., \& Johnson, P. (1987). Accommodation theory: The last decade and beyond. In M. L. Mclaughin (Ed.), Communication yearbook 10 (pp. 13-48). Newbury Park, CA: Sage.

Giles, H., \& Smith, P. M. (1979). Accommodation Theory: Optimal levels of convergence. In H. Giles \& R. N. St. Clair (Eds.), Language and social psychology (pp. 45-65). Oxford, UK: Blackwell.

Giles, H., Taylor, D., \& Bourhis, R. (1973). Towards a theory of interpersonal accommodation through language: Some Canadian data. Language in Society, 2, 177-192.

Goodwin, C. (1986). Between and within: Alternative sequential treatments of continuers and assessments. Human Studies, 9, 205-217.

Heinz, B. (2003). Backchannel responses as strategic responses in bilingual speakers' conversations. Journal of Pragmatics, 35, 1113-1142.

Kollock, P., Blumstein, P., \& Schwartz, P. (1985). Sex and power in interaction: Conversational privileges and duties. American Sociological Review, 50, 34-46.

Li, H. Z. (1999a). Communicating information in conversations: A cross-cultural comparison. International Journal of Intercultural Relations, 23, 387-409. 


\section{H. Z. $\mathrm{Li}$}

Li, H. Z. (1999b). Grounding and information communication in inter- and intra-cultural dyadic discourse. Discourse Processes, 28(3), 195-215.

Li, H. Z. (2001). Co-operative and intrusive interruptions in inter- and intra-cultural dyadic discourse. Journal of Language and Social Psychology, 20(3), 259-284.

Li, H. Z. (2004). Gaze and mutual gaze in inter- and intra-cultural conversation. International Journal of Language and Communication, 20, 3-26.

Liu, J. H. (1987). Denwa de no aizuchi hindo no chuunichi hikaku [Chinese-Japanese comparison of the frequency of backchannels over the phone]. Gengo [Language], 16(12), 93-97.

Mizuno, Y. (1988). Chuugokugo no aizuchi [Chinese backchannels]. Nihongogaku [Japanese Language Studies], 7(12), 18-23.

Roter, D. J., \& Hall, J. A. (1992). Doctors talking with patients/patients talking with doctors: Improving communication in medical visits. Westport, CT: Auburn House.

Schegloff, E. A. (1982). Discourse as an interactional achievement: Some uses of "uh huh" and other things that come between sentences. In D. Tannen (Ed.), Analyzing discourse: Text and talk (Georgetown University Round Table on Languages and Linguistics) (pp. 71-93). Washington, DC: Georgetown University Press.

Tao, H. Y., \& Thompson, S. A. (1991). English backchannels in Mandarin conversations: A case study of superstratum pragmatic "interference". Journal of Pragmatics, 16, 209-233.

Yngve, V. H. (1970). On getting a word in edgewise. In Papers from the sixth regional meeting, Chicago Linguistic Society (pp. 567-578). Chicago: Chicago Linguistic Society.

\section{Appendix A: Examples of Backchannel Responses}

Excerpt 1 (Code-switching, overlapping)

Dr.: I suggest that you take an/X-ray/, then you may rest/assured/.

Pt.: /OK/ /hao,haol

Pt.: Even thunder cannot wake/me up/

Dr.: /OK/

Excerpt 2 (Nod with uhm, non-overlapping)

Dr.: Yesterday afternoon I developed some chest pain.

Pt.: Uhm

Excerpt 3 (Nod, overlapping)

Pt.: What happened was last week I went swim/ming/.

Dr.: /Nod/

Excerpt 4 (Nodding with yeah, overlapping)

Pt.: I just took some codei/ne/.

Dr.: /Yeah/

Excerpt 5 (OK, non-overlapping)

Dr.: OK. I'd like to give you some test.

Pt.: OK. 
Excerpt 6 (Uhm paying attention, overlapping)

Dr.: These side effects are not that com/mon./

Pt.:

/Uhm/

Excerpt 7 (Oh showing surprise, overlapping)

Dr.: So what's your problem?

Pt.: I have chest pain.

Dr.: Oh!

Pt.: I'm kind of scared. A friend of mine's daughter died of chest pain when swimming.

Dr.: Oh!

Excerpt 8 (Nod with Right, overlapping)

Dr.: If you take too much, that is harmful/to you/

Pt.:

/right/

Excerpt 9 (Code-switching, overlapping)

Dr.: Feeling any better now?

Pt.: A little bit better after seeing the/doctor/

Dr.: /Yeah/

Excerpt 10 (Yeah, overlapping)

Dr.: How is your appetite generally?

Pt.: I think it'/s good/

Dr.: /Yeah/

Excerpt 11 (OK, overlapping)

Dr.: How about your dad? Is Dad still living?

Pt.: Oh, ye/s/

Dr.: $\quad / O K /$

Excerpt 12 (Uhm with agreement, non-overlapping)

Dr.: Has any of your family members, like brothers, sisters, father, had chest pain before?

Pt.: Uhm. My mother also suffered from chest pain.

Dr.: Do you have any questions at all?

Pt.: Yeah. I was just wondering why you prescribe codeine. I thought it was my heart that had a problem. That's why I had chest pain, right?

Dr.: Uhm.

Excerpt 13 (Nod with I see, non-overlapping)

Dr.: I gave this medicine to some other patients, and they felt good.

Pt.: I see. 
116 H. Z. $\mathrm{Li}$

Excerpt 14 (Repetition, non-overlapping)

Dr.: So what's the problem?

Pt.: I had chest pain.

Dr.: When did it happen?

Pt.: Yesterday.

Dr.: Yesterday.

Excerpt 15 (Uhm with understanding, non-overlapping)

Dr.: This medicine will have some side effects.

Pt.: Uhm.

Pt.: Shall I take the medicine with meals?

Dr.: Just take it with water

Pt.: Uhm.

Excerpt 16 (Shi Ma, non-overlapping)

Pt.: Probably because I didn't sleep well the night before last.

Dr.: Shi Ma [Is that so]?

Excerpt 17 (Shi Zhe Yang Zi with a flat and falling tone, non-overlapping)

Pt.: It is a little bit difficult for me to walk up and downstairs.

Dr.: Shi Zhe Yang $\mathrm{Zi}$ [Is that really so].

\section{Appendix B: The Listener Recall Test Questions (Dialogue 1)}

When answering the following questions, please provide as many details as you can. That is, write down anything relevant to the questions.

1. Why did the patient come to see you?

2. When did the patient's symptoms start?

3. How did the patient sleep last night?

4. What other disease does the patient have, and how does it affect the patient's daily life?

5. What was the main reason the patient went swimming?

6. When (how many years ago) did the patient have similar symptoms before?

7. When the patient had similar symptoms before, what was done?

8. According to the patient, what did his/her mother die of?

-Please write down anything else you want to add. 